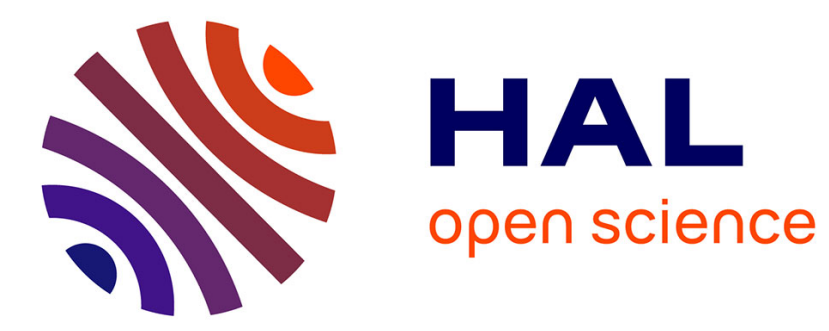

\title{
Ultrasound Backscatter characterization by using Markov random field model
}

Nizar Bouhlel, Sylvie Sevestre-Ghalila, Mériem Jaidane, Christine Graffigne

\section{To cite this version:}

Nizar Bouhlel, Sylvie Sevestre-Ghalila, Mériem Jaidane, Christine Graffigne. Ultrasound Backscatter characterization by using Markov random field model. IEEE International Conference on Acoustics, Speech and Signal Processing, May 2006, Toulouse, France. pp.1124-1127. hal-00173925

\section{HAL Id: hal-00173925 \\ https://hal.science/hal-00173925}

Submitted on 21 Sep 2007

HAL is a multi-disciplinary open access archive for the deposit and dissemination of scientific research documents, whether they are published or not. The documents may come from teaching and research institutions in France or abroad, or from public or private research centers.
L'archive ouverte pluridisciplinaire HAL, est destinée au dépôt et à la diffusion de documents scientifiques de niveau recherche, publiés ou non, émanant des établissements d'enseignement et de recherche français ou étrangers, des laboratoires publics ou privés. 


\title{
ULTRASOUND BACKSCATTER CHARACTERIZATION BY USING MARKOV RANDOM FIELD MODEL
}

\author{
BOUHLEL N. ${ }^{(1,2)}$, SEVESTRE-GHALILA S. ${ }^{(1)}$,JAIDANE M. ${ }^{(2)}$ and GRAFFIGNE C. ${ }^{(1)}$ \\ (1)Université René Descartes, Laboratoire MAP5 \\ 45, Rue des Saints Pères, Paris 75006, France \\ (2) Ecole Nationale d'Ingénieurs de Tunis, Unité Signaux et Systèmes \\ Campus Universitaire Tunis1002, Tunisia
}

\begin{abstract}
This paper evaluates a $\mathcal{K}$-Markov random field model for retrieving information about backscatter characteristics, especially regularity spacing scatterers in simulated ultrasound image. The model combines a statistical $\mathcal{K}$-distribution that describes the envelope of backscattered echo and spatial interaction given by Markov random field (MRF). Parameters estimated by the conditional least squares (CLS) estimation method on simulated radio-frequency (RF) envelope image show that the interaction parameters measure the degree of the randomness of the scatterers.
\end{abstract}

\section{INTRODUCTION}

Many researchers have used statistical models to describe the envelope of the backscattered echo from tissues called the radio-frequency (RF) envelope. The parameters of these distributions indicate characteristics such as density (the number of scatterers within the resolution cell of the transducer, where scatterers are defined as small structures in tissue, reflecting and scattering the incoming wave), and scatterer amplitude related to the size of the scatterers. Different backscatter models are proposed. The Rayleigh model is commonly employed [1], but it requires conditions, such as the presence of large number of randomly located scatterers. The $\mathcal{K}$-distribution has been introduced as a valid model to represent these statistics and to provide better fit to data $[2,3]$. It has also potential clinical importance in tissue characterization [2, 4].

In addition to density and scatterer amplitude, another important backscatter characteristic is scatterer spacing, or regularity [5]. Regularity quantifies how both randomly and regularly spaced scatterers are present in the tissue.

The parameters of $\mathcal{K}$-distribution can't inform about the regularity of scatterers, because the shape parameter of $\mathcal{K}$, also called the effective density, inform only about the density and the scatterer amplitude [6]. We found in literature, several attempts to represent the regularity by spatial models like Markov random field (MRF). It can be divided into two categories: the first one, tries to model the ultrasound image using Gaussian MRF. This latter, can't respect the statistical model of the envelope of the backscattered echo. The second one segments the image using a prior model which does not integrate any information about texture present in the image $[7,8]$. So, we propose to use a $\mathcal{K}$-Markov random field model [9], which locally guaranties better fit than classic models, such as the Gaussian Markovian model. The main difference compared to the classical MRF model is that our model is based on the $\mathcal{K}$-distribution. To evaluate and understand the parameters role in the model, we use an ultrasound
RF simulator that realistically models the physical process in RF signal generation, and uses the density, spacing and the amplitudes to describe the scattering process.

The paper is organized as follows. First, we introduce some backscatter characteristics. Second, we present the $\mathcal{K}$-MRF model and its features. Parameters estimation method based on Conditional Least Square (CLS) follows. Experiments with a realistic Ultrasound (US) RF simulator $[10,11]$ are described next, followed by results of estimation MRF parameters by CLS and link with the backscatter characteristics regularity. Analysis, discussion, and concluding remarks close up the paper.

\section{BACKSCATTER CHARACTERISTICS}

The three backscatter characteristics are density, spacing, and scatterer cross section (scatterer amplitude). Density is a measure of the average number of scatterers in the resolution cell of the US transducer. Spacing (or placement) refers to the randomness or regularity of the distances between scatterers. The scattering amplitudes or scattering cross section show the amplitude variations caused by many phenomena such as attenuation, absorption, diffraction, etc...

Some RF simulators take into account these characteristics and consider them stochastic. Indeed, the simulator given in $[10,11]$ takes into account the randomness of the amplitudes by the signal to noise rate, $S N R_{a}$ of the scattering cross-section. In this case, the amplitudes are gamma-distributed with shape parameter $a^{2}$ and with unit scale parameter. The scatterer spacing was also characterized. In [5], the gamma distribution has been shown to accurately describe scatterer spacing. By denoting the mean spacing between scatterers as $\bar{x}$, the spacing distribution is described by the gamma distribution, $\gamma(u, v)$, with shape parameter $u$ and scale parameter $v=\bar{x} / u$. For large $u$, the spacing is regular. For $u=1$ the scatterers are randomly spaced (diffuse). When $u<1$, the scatterers are clustered [5]. Figure (1) illustrates this behavior for various values of $u$ when the mean inter-scatterer distance $\bar{x}$ is maintained at unity.

\section{RF ENVELOPE IMAGE SIMULATOR}

The ultrasound RF simulator introduced in $[10,11]$ is used to generate echo envelopes. This simulator realistically models the actual physical process in RF signal generation, and uses the density $(N)$, spacing $(u)$, and amplitudes $\left(S N R_{a}\right)$ to describe the scattering process. The parameters for the simulator are as follows: $f_{0}$ (center frequency) $=3.5 \mathrm{MHz}, B$ (bandwidth) $=0.8 \mathrm{MHz}, v$ (velocity of sound $)=1446 \mathrm{~m} / \mathrm{s}$, sampling window size $=3,7 \mathrm{~cm}$. In the simulation, the RF backscattered signal consisting of 100 A lines (1-D 


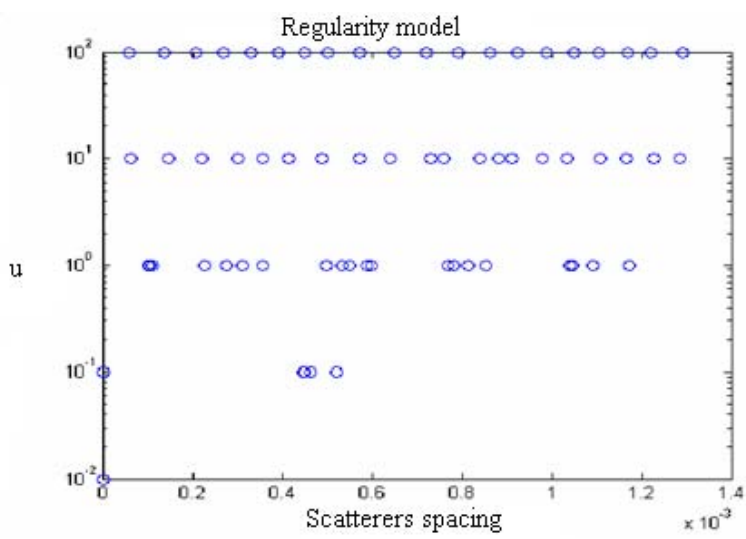

Fig. 1. Spatial scatterer organization with variation of $u$

RF signals) was sampled at $40 \mathrm{MHz}$. Therefore, we obtained 2048 samples in each line. To ensure samples uncorrelated, every second sample in every A line is used. The received RF backscattered signal is demodulated at $f_{0}$ using the setup shown in figure (2), resulting in inphase and quadrature components $\mathrm{X}$ and $\mathrm{Y}$, respectively. A 10th order $2 \mathrm{MHz}$ Butterworth lowpass filter is applied to both components, and the echo envelope is computed as $\sqrt{X^{2}+Y^{2}}$ [11]. We

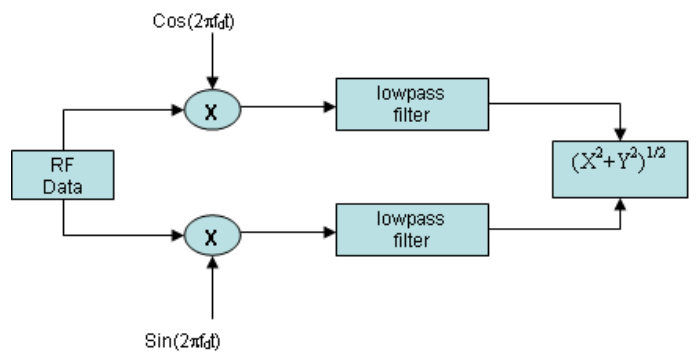

Fig. 2. Block diagram of the processing path from RF signal to the envelope of the signal.

will explore along this paper some of the scattering conditions undertaking simple simulations. The resulting backscattered signal envelope is used for statistical processing. A typical histogram obtained through this trial is shown. As will be shown later, the resulting envelope will correspond to $\mathcal{K}$-distribution.

\section{4. $\mathcal{K}$-MRF MODEL}

\subsection{Statistical model: $\mathcal{K}$ distribution}

The backscattered ultrasonic echo from tissue can be described in terms of $\mathcal{K}$-distribution. The two parameters of this distribution, together provide information on the number density of the scatterers, the variation in the scattering amplitudes within the range cell and the mean scattering amplitudes.

The probability density function of the amplitude $A$ of the RF envelope, which fits $\mathcal{K}$-distribution with parameters $(\alpha, \beta) \in\left(\mathbb{R}_{+}^{*}\right)^{2}$, denoted as $\mathcal{K}(\alpha, \beta)$, is given by:

$$
f(A)=\frac{2 \beta}{\Gamma(\alpha)}\left(\frac{\beta A}{2}\right)^{\alpha} K_{\alpha-1}(\beta A) ; \quad \forall A \in \mathbb{R}_{+},
$$

here $\Gamma($.$) is Gamma function, K_{\alpha-1}$ is the modified Bessel function of the second kind of order $(\alpha-1), \alpha$ is the shape parameter and $\beta$ is the scaling parameter of the $\mathcal{K}$-distribution. The parameter $\alpha$ is also called the effective number of scatterers in the resolution cell and should be estimated from the moments of the $\mathcal{K}$-distribution. The parameter $\alpha$ can be related to the actual density of scatterers $N$, as:

$$
\alpha=N(1+\gamma), \quad \gamma>-1
$$

where $\gamma$ is parameter describing the lack of uniformity of the scattering cross sections in the range cell. The authors of [12] proved that $\alpha$ informs about the homogeneity of tissues. So, their conclusions were used to characterize and localize normal and abnormal regions containing a cancer.

The $\mathcal{K}$-distribution is not able to take into account the regularity spacing between reflectors. As, the example in figure (5.a) shows, this failing can then lead to parameter $\alpha$ almost similar for simulations of signals RF realized from different values for the couple of parameters $\left(S N R_{a}, u\right)$. A generalized model based on Markov random field is here proposed. This model can characterize the envelope of the backscattered echo from a range of scatterers with varying number densities, varying scatterer amplitudes and varying scatterer spacing.

\subsection{Spatial Model: $\mathcal{K}$-MRF Presentation and Features}

Our goal is to represent the RF envelope image with spatial model based on the $\mathcal{K}$-distribution as the probability distribution function of the observed amplitudes of the envelope image. We use the Markov random field tool which allows taking into account the spatial information. So, we suppose that at each pixel $s$ of pixel set $S$ of the image, the envelope amplitude $A_{s}$ given $A_{V_{s}}=\left(A_{r}\right)_{r \in V_{s}}$ of the pixels of the neighbourhood $V_{s}$, follows $\mathcal{K}$-distribution with parameters depending on $A_{V_{s}}$. The construction of our Markovian model is given in [9]. The Markov random field for the observed ultrasound image $A$ in which the conditional probability distribution function of $A_{s}$ knowing the neighbourhood $A_{V_{s}}=\left(A_{r}\right)_{r \in V_{s}}$ is a $\mathcal{K}$-distribution with parameters $\left(\alpha_{s}, \beta\right)$ defined as follows:

$$
\left(A_{s} / A_{r}, r \in V_{s}\right) \propto \mathcal{K}\left(\alpha_{s}, \beta\right)
$$

Where

$$
\alpha_{s}=a_{s}+1+\sum_{r \in V_{s}} b_{s r} \ln A_{r}, \quad \beta>0
$$

$\left(a_{s}, b_{s r}, \beta\right)$ are the parameters of the model. The first and the second moment of $\mathcal{K}$-MRF are given by:

$$
\left\{\begin{array}{l}
E\left\{A_{s} / A_{r}, r \in V_{s}\right\}=\frac{\Gamma\left(\alpha_{s}+0.5\right)}{\Gamma\left(\alpha_{s}\right)} \frac{2}{\beta} \Gamma(1.5) \\
E\left\{A_{s}^{2} / A_{r}, r \in V_{s}\right\}=4 \frac{\alpha_{s}}{\beta^{2}}
\end{array}\right.
$$

In order to understand the role of the parameters of our Markovian model based on $\mathcal{K}$-distribution, some simulations have been done in [13]. In what follows, we consider the field stationnary of order 1 or 2. Therefore, the parameters are constant through the considered site and are noted:

$$
b_{s r}=b_{i}, \quad i \in\{1,2,3,4\} \quad \forall r \in V_{s} .
$$

Where $b_{1}$ (resp. $b_{2}$ ) presents the interaction of two neighboring pixels on the horizontal direction (resp.vertical direction) and $b_{3}, b_{4}$ respectively on the first and the second diagonal.

Due to its computation efficiency, the Conditional least squares estimate (CLS) method has been commonly accepted to estimate the parameters of our models. It minimizes the quadratic difference between the central pixel $A_{s}^{2}$ and its neighbors. 


\section{EVALUATION ON SIMULATED RF ENVELOPE IMAGE}

We use the RF simulator described above to generate echo envelope with different configurations of the triplet $\left(N, S N R_{a}, u\right)$. Our goal is to evaluate the ability of our MRF model to characterize the spacing of reflectors compared to no spatial $\mathcal{K}$-distribution. We consider three different examples, in second and third example, ten simulations have been done. We suggest through these examples, first, to illustrate the contribution of the model proposed in this paper to characterize the spacing of reflectors, second, to show the inability of $\mathcal{K}$-distribution to characterize the scatterer spacing and finally, to show the similarity between the parameter $a_{s}$ of our model and the parameter $\alpha$ of the $\mathcal{K}$-distribution.

\subsection{Evaluation Methods}

First example: we generate different scatterer spacing values $u$, but we maintain constant the density of reflectors $N=50$ and the value $S N R_{a}=1$ of the scattering cross-section. This particular case is done to show the influence of scatterers repartition and regularity on our spatial model. We generate three regions corresponding to three values of $u \in\{0.2,1,10\}$. Figure (3) shows these regions. The envelope histograms of region (a) and (c) show specific probability density functions. Figure (4.a) shows the histogram for region (a), the shape appears to have a $\mathcal{K}$-distribution form. Figure (4.b) shows the histogram of region (c). The shape appears to have a Rayleigh form. These observations about envelope distributions are consistent with those of other studies $[2,4]$. To show the ability of our

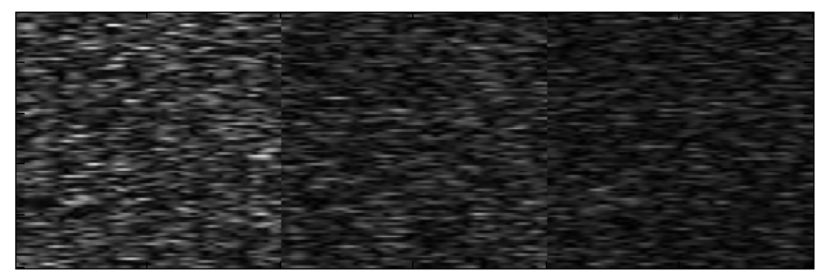

(a)

(b)

(c)

Fig. 3. Three types of textures for three various organizations: region (a): less regular, $u=0.2$, region (b): $u=1$, region (c): more regular, $u=10$.

spatial model, we use the model parameters as textural features for evaluation of scatterers regularity. Briefly, we apply the model in each region of the simulated RF envelope image and we estimate the parameters by the CLS method mentioned above. The table (1) shows the parameters estimated for the three regions: (a),(b) and (c). For comparison, we notice that the values of $a_{s}$ and $\beta$ increase from region (a) to region (c). This is due to homogeneity of the regions. We note that horizontal interaction is mentioned by $b_{1}$, because the chosen RF simulator excludes all interactions except the horizontal one. That's why, the others $b_{i}, i \in\{2,3,4\}$ are very weak. From region (a) to (c), the interaction $b_{1}$ increases due to the regularity of the spacing between reflectors.

Second example: The mean number of scatterers per cell is fixed, $N=20$. We generate two series of 10 simulations for the following both cases $\left(S N R_{a}=0.8, u=5\right)$ and $\left(S N R_{a}=1.4, u=\right.$ 0.25 ). Figure (5.a) shows the value of $\alpha$ estimated from empirical moments of the $\mathcal{K}$-distribution for each simulations. This graph

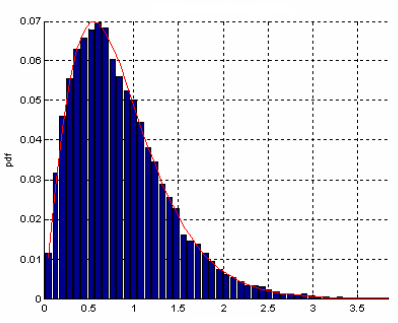

(a) $\alpha=3.13, \beta=3.54$

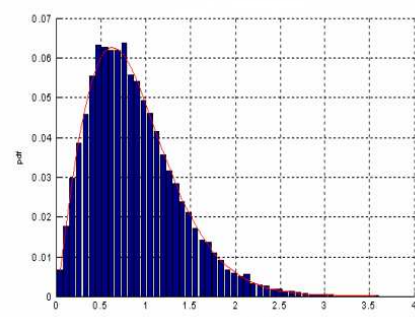

(b) $\alpha=6.37, \beta=5.05$
Fig. 4. The histograms of simulated echo envelope for region (a) (figure 3.a) and region (c) (figure 3.c) are compared with the density function of $\mathcal{K}$-distribution. The parameters of the distribution $(\alpha, \beta)$ are given for each region.

\begin{tabular}{|l|c|c|c|c|c|c|}
\hline & $a_{s}$ & $b_{1}$ & $b_{2}$ & $b_{3}$ & $b_{4}$ & $\beta$ \\
\hline \hline Region (a) & 4.66 & 2.04 & 0.17 & -0.20 & -0.02 & 3.54 \\
\hline Region (b) & 6.16 & 2.80 & 0.04 & -0.12 & -0.03 & 4.14 \\
\hline Region (c) & 9.12 & 4.18 & -0.29 & 0.20 & 0.10 & 5.05 \\
\hline
\end{tabular}

Table 1. Parameters of the $\mathcal{K}-\mathrm{MRF}$ estimated by CLS on the 3 regions displayed in figure 3 .

shows several overlappings of the two case of curves. That's implies similar values of $\alpha$. On the other hand, the application of the spatial model elaborated, allows, for this example, to distinguish scatterer spacing and representing them. Figure (5.b) shows the measure of the first interaction parameter $b_{1}$ applied to the previous simulations. The values of $b_{1}$ are indicators in the way that are distributed reflectors. For a regular spacing $u=5$, the interaction is strong and $b_{1}$ is important. For irregular spacing, $u=0.25$, the parameter $b_{1}$ is weak. This justifies the fact that there is no overlapping of the two curves.

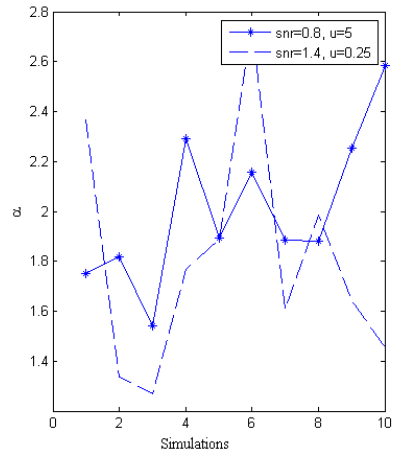

(a)

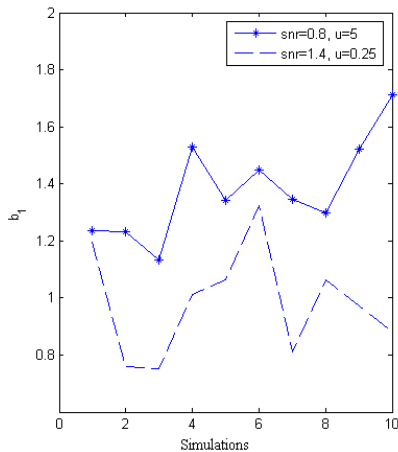

(b)
Fig. 5. (a)Estimation of the effective number of scatterers $\alpha$ in the resolution cell. (b) Estimation of parameter $b_{1}$ of our MRF model. Estimation of $\alpha$ and $b_{1}$ have been done for a couple of simulation $\left(S N R_{a}=0.8, u=5\right)$ and $\left(S N R_{a}=1.4, u=0.25\right)$.

Third example: In this example $N=20$ and $u=0.25$, while using two different scattering amplitudes $S N R_{a}=1$ and 
$S N R_{a}=1.4$. Figure (6.a) shows the estimation values of $b_{1}$ for these configurations. We conclude that the curves overlap. This is due to the use of the same value of $u$. Figure(6.b) and (6.c) show the estimation values of $\alpha$ and $a_{s}$ parameter of our Markovian model. We observe that the estimated parameter $a_{s}$ is proportional to the estimated value of $\alpha$. Therefore, this similarity implies that $a_{s}$ parameter should be related to the effective number of the scatterers.

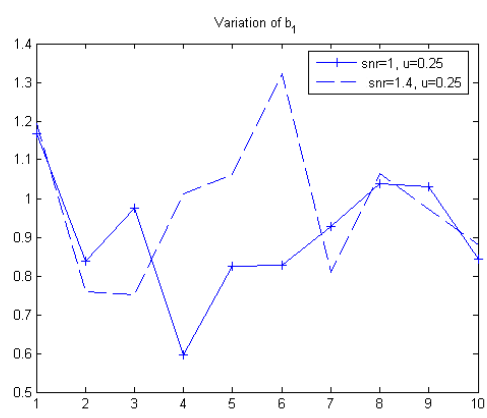

(a)

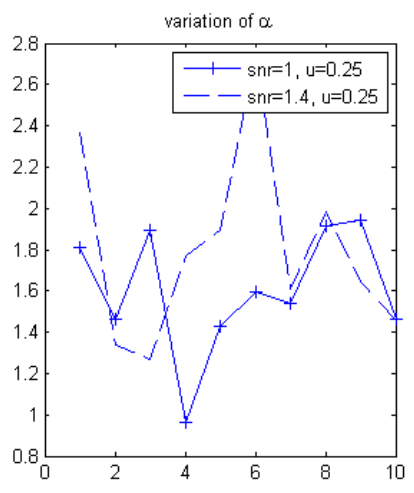

(b)

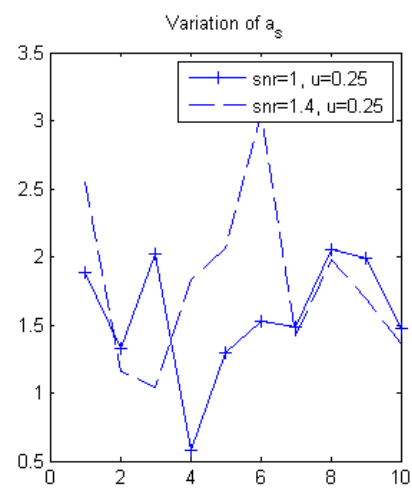

(c)
Fig. 6. (a) and (c) Estimation of the interaction parameter $b_{1}$ and $a_{s}$ for $\mathcal{K}$-MRF model. (b) Estimation of $\alpha$ for $\mathcal{K}$-distribution. All the estimation have been done for the configurations $\left(S N R_{a}=1, u=\right.$ $0.25)$ and $\left(S N R_{a}=1.4, u=0.25\right)$.

\subsection{Results}

For the first example, it is clear that the value of $\mathcal{K}$-distribution parameter $\alpha$ gives information about the homogeneity of region but not the direction of the interactions. The interaction parameters of our model is able to take into account this effect. In the second example, the interaction parameter $b_{i}$ of our model permits to distinguish between spacing regularity for scatterers volume that have the same $\alpha$. In the final example, the information given by $a_{s}$ is the same that $\alpha$.

\section{CONCLUSION AND DISCUSSION}

A spatial Markov random field model is used to characterize ultrasound backscatter. Experimental results on simulated RF envelope image show the behavior of every parameter of the model. We notice that the parameter of our MRF model $a_{s}$ follows the value of effective number of the scatterers $\alpha$ and so, we preserve the properties of the statistical model: $\mathcal{K}$-distribution. For the interaction parameters of the MRF model $b_{i}$, it indicates the degree of regularity of scatterers. So, for a regular spacing, the interaction is strong and $b_{i}$ is important, and for irregular spacing, the parameter $b_{i}$ is weak.

\section{REFERENCES}

[1] J.W. Goodman, "Statistical properties of laser speckle patterns," J.C. Dainty (Ed.), Laser Speckle and Related Phenomena, 2nd Edition, Springer, Berlin,, vol. 9 of Topics in Applied Physics, pp. 976, 1984.

[2] J.M. Reid F. Forsberg E.J. Halpern C.W. Piccoli R. C Molthen, P.M. Shankar and B.B. Goldberg, "Comparisons of the rayleigh and k-distribution models using in vivo breast and liver tissue," Ultrasound Med. Biol., vol. 24, no. 1, pp. 93100, 1998.

[3] P. M. Shankar, V. A. Dumane, J. M. Reid, V. Genis, F. Forsberg, C.W. Piccoli, and B.B Goldberg, "Use of the k-distribution for classification of breast masses," Ultra. Med. Biol., vol. 26, no. 9, pp. 1503-1510, 2000.

[4] P. M. Shankar, "A model for ultrasonic scattering from tissues based on the k-distribution," Phys. Med. Biol., vol. 40, pp. 1633-1649, 1995.

[5] Robert M. Cramblitt and Kevin J. Parker, "Generation of nonrayleigh speckle ditribution using marked regularity models," IEEE Trans. On Ultrasonics, Ferro. And Frequency Control, vol. 46, no. 4, pp. 867-873, July 1999.

[6] E. Jakeman and P. N. Pusey, "A model for non-rayleigh sea echo," IEEE Trans. On antennas and Prop., vol. 24, no. 6, pp. 806-814, november 1976.

[7] C. Hass, H. Ermert, S. Holt, P. Grewe, A. Machraoui, and J. Barmeyer, "Segmentation of 3d intravascular ultrasonic images based on random field model," Ultrasound in Med.and Biol, vol. 26, no. 2, pp. 297-306, 2000.

[8] G. Xiao, M. Brady, J. A. Noble, and Y. Zhang, "Segmentation of ultrasound b-mode images with intensity inhomogeneity correction," IEEE Trans. on Medical Imaging, vol. 21, no. 1, January 2002.

[9] Bouhlel N., Sevestre G. S., Rajhi H., and Hamza R., "New markov random field model based on k-distribution for textured ultrasound image," Medical Imaging 2004: Ultrasonic Imaging and Signal Processing, SPIE International Symposium, vol. 5373, pp. 363-372, 14-19 February 2004.

[10] P. Mohana Shanker, "A general statistical model for ultrasonic backscattering from tissues," IEEE, Trans. On Ultrasonics, Ferro. And Frequency Control, vol. 47, no. 3, pp. 727-736, May 2000.

[11] V. Manoj Narayanan, P.M. Shanker, and John M. Reid, "Nonrayleigh statistics of ultrasonic backscattered signals," IEEE, Trans. On Ultrasonics, Ferro. And Frequency Control, vol. 41, no. 6, pp. 845-852, November 1994.

[12] Ortega H. Picolli C.W. Goldberg B.B Shankar P.M., Reid J.M., "Use of non-rayleigh statistics for identification of tumors in ultrasonic b-scans for the breast," IEEE Trans. On Medical Imaging, vol. 12, no. 4, pp. 687-692, 1993.

[13] Bouhlel N., Sevestre G. S., Rajhi H., and Hamza R., "Nouveau modèle de texture markovien basé sur la loi $\mathrm{k}$ : Application l'échographie," GRETSI 03, vol. 1, pp. 112-115, 8-11 september 2003. 